\title{
A Low-Power and In Situ Annealing Technique for the Recovery of Active Devices After Proton Irradiation
}

\author{
Laurent A. Francis, Amor Sedki, Nicolas André, Valéria Kilchytska, Pierre Gérard, Zeeshan Ali, \\ Florin Udrea, and Denis Flandre.
}

\begin{abstract}
In this paper, we study the recovery of onmembrane semiconductor components, such as N-type Field-Effect Transistors (FETs) available in two different channel widths and a Complementary Metal-OxideSemiconductor (CMOS) inverter, after the exposure to high dose of proton radiation. Due to the ionizing effect, the electrical characteristics of the components established remarkable shifts, where the threshold voltages showed an average shift of $-480 \mathrm{mV}$ and $-280 \mathrm{mV}$ respectively for $6 \mu \mathrm{m}$ and $24 \mu \mathrm{m} \mathrm{N}$-channel transistors, likewise the inversion point of the inverter showed an important shift of $-690 \mathrm{mV}$. The recovery concept is based mainly on a micro-hotplate, fabricated with backside MEMS micromachining structure and a Silicon-On-Insulator (SOI) technology, ensuring rapid, low power and in situ annealing technique, this method proved its reliability in recent works. Annealing the $\mathrm{N}$-channel transistors and the inverter for $\mathbf{1 6} \mathrm{min}$ with a temperature of the heater up to $385^{\circ} \mathrm{C}$, guaranteed a partial recovery of the semiconductor based components with a maximum power consumption of $66 \mathrm{~mW}$.
\end{abstract}

Keywords - Field Effect Transistors (FETs), In situ annealing, Micro-hotplates, Proton radiation, Radiation mitigation.

\section{INTRODUCTION}

Tharc he Silicon-On-Insulator (SOI) technology is one favored hardening method for circuits exposed to natural radiations, in particular for single event effects (SEE) [1], in addition to the tremendous advantages and great resistivity at high temperatures up to $300{ }^{\circ} \mathrm{C}$, making it a very suitable technology in harsh environments [2]. However, this technology still remains sensitive to total ionizing doses (TID) [3], where increased densities of both oxide-trapped charges and interface traps lead to massive degradations in transistor's fundamental parameters (threshold voltage, carriers mobility, leakage currents, etc.). The recovery of transistors after their irradiation can be guaranteed by the neutralization of positive trapped charges helped by thermal annealing or a tunneling effect under

L.A. Francis, S. Amor, N. André, V. Kilchytska, P. Gérard, and D. Flandre are with the Institute of Information and Communication Technologies, Electronics, and Applied Mathematics, Université catholique de Louvain, B1348 Louvain-la-Neuve, Belgium (e-mail: \{laurent.francis\}, \{nicolas.andre $\},\{$ valeria.kilchytska \}, \{pierre.gerard\}, \{denis.flandre\}@uclouvain.be, and sedkiamor@gmail.com). L.A. Francis is corresponding author. external electrical field. The thermal annealing process consist in the exposure of radiated devices to high temperature between 300 and $400{ }^{\circ} \mathrm{C}$ for about one hour [4], otherwise by long term stability at room temperature [5]. The latter approach is efficient but constrained by the post-CMOS thermal budget, limited by e.g. the thermally activated dopant diffusion or the degradation of the metal layers, a wide variety of reliability issues related to the different coefficients of thermal expansion for System-in-Package (SiP) technology, and by the bulky equipments and their high power consumption needed to achieve the thermal treatment. Recent works improved a revolutionary success by improving an in situ low power total recovery of semiconductor based devices after high gamma dose irradiation [6]. In this work, our interest is rather focusing on the proton radiation, by studying the electrical characteristics of SOI electronic components embedded in a $5 \mu \mathrm{m}$-thick microhotplate membrane and analyzing the degradations that appeared after proton irradiation. As well we are investigating the recovery of the components by thermal annealing up to $385{ }^{\circ} \mathrm{C}$. To do so we used a special MEMS structure based on micro-hotplates, which cluster the studied electronic components and the micro-heater on a single membrane, for better power conservation and integration efficiency, where the temperature is monitored by an integrated thermo-diode as demonstrated in [7].

The first part of this paper, describes the used device's structure, specify its fabrication technology, demonstrate the used characterization technique and details the stabilization method, including the description of thermo-diode's calibration methodology. The second section, reports the applied referential characterization of the components, present the proton irradiation process of the device and study the related degradations of the semiconductor based components. The third and last section discusses the applied thermal annealing process, as well, evaluates the partial recovery of the electrical characteristics of the MOSFET based components and the CMOS inverter.

F. Udrea is with the Department of Engineering, Electrical Engineering Division, University of Cambridge, $9 \mathrm{JJ}$ Thomson Avenue, CB3 0FA, Cambridge, United Kingdom (e-mail: florin.udrea@ucam.uk).

S.Z. Ali and F. Udrea are with Cambridge CMOS Sensors Ltd, Deanland House, Cowley Road, Cambridge, CB4 0DL, United Kingdom, (e-mail: zeeshan.ali@ccmoss.com). 


\section{EXPERIMENTAL PROCEDURE}

\section{A. Device's technology and description}

The used technology to fabricate the MEMS structure is $0.1 \mu \mathrm{m}$ Partially Depleted (PD) SOI (XI.10 from X-FAB, Erfurt, Germany). The device is based mainly on a microhotplate, obtained by a post-CMOS Deep Reactive Ion Etching (DRIE) of the $400 \mu \mathrm{m}$ thick silicon layer. The backside etching stops when reaching the buried $1 \mu \mathrm{m}$ thick $\mathrm{SiO}_{2}$ layer to form a circular membrane with a diameter of $600 \mu \mathrm{m}$. On the front side the membrane is centered by a Joule heater with $200 \mu \mathrm{m}$ diameter, made by a $0.5 \mu \mathrm{m}$ thick tungsten layer. Two $\mathrm{N}$ channel transistors, one CMOS inverter and one PIN diode are placed on the membrane around the micro-heater (see Figure. 1), whereas the membrane and the electronic component's temperature is monitored by the PIN diode. The benefit of the membrane consists mainly in its low thermal dissipation, which allow the micro-hotplates to reach high temperatures with good linearity, in addition to its high resistivity to the ionizing radiations making this technique very suitable in harsh environments [7].

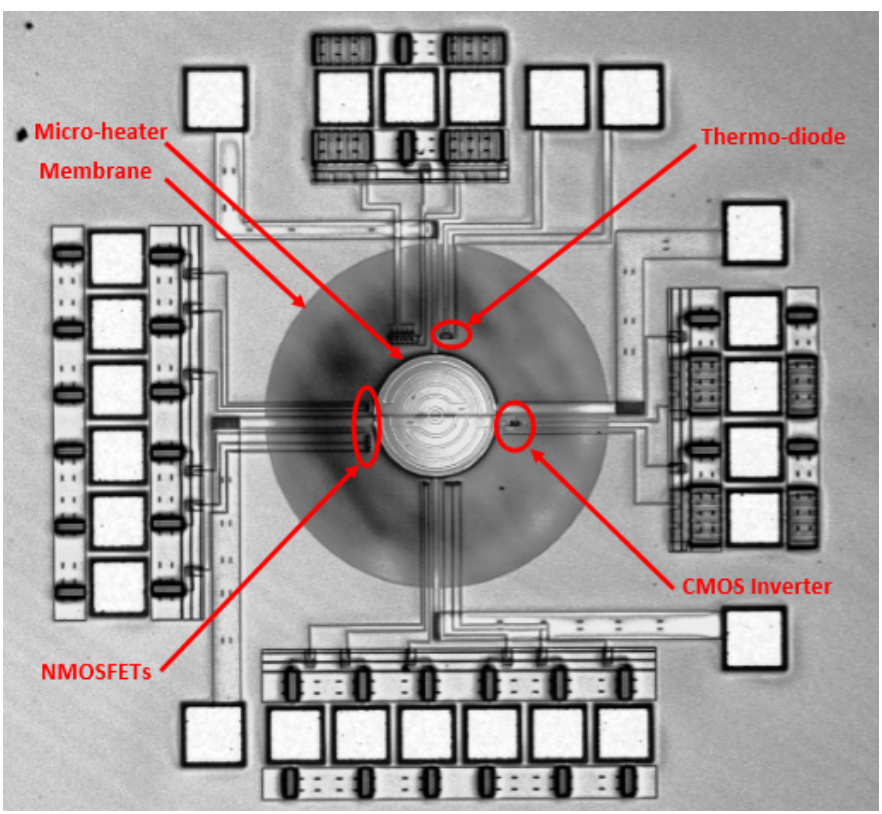

Fig. 1. Optical micrograph of the front view of the MEMS device, presenting the location of the studied on-membrane embedded components.

To avoid electrical parasites during measurements the device was packaged in a Dual In Line (DIL) ceramic package where the metallic pads were electrically connected to the 24 pins by wire-bonding, in respect to a pre-fixed order. After preparing the device, it is inserted in a Zero Insertion Force (ZIF) support, then connected to the triaxial ports of a test fixture box. The device is connected to a Semiconductor Parameter Analyzer (HP4145) to be controlled by IC-CAP software through an adjustable interface.

After the backside micromachining, the electrical characteristics of the MOSFET based components showed a significant instability in the I-V curves, this returns mainly to the creation of parasitic charges during the engraving of the silicon substrate to create the membrane. Thus, a pre- stabilization process is considered mandatory to stabilize the characteristics of the MOSFETs before starting the characterization part. In order to neutralize the trapped charges, the process consists in annealing the MOSFETs by 4 successive cycles of $30 \mathrm{~s}$ each, where the micro-hotplate's temperature is gradually increased up to $265^{\circ} \mathrm{C}$ by supplying the micro-heater with the corresponding electrical power. Subsequently, the onmembrane semiconductor components showed as well a reliable characteristics after high temperature annealing up to $335^{\circ} \mathrm{C}$. A deep study thermal characteristics of the different devices is available in [8].

\section{B. Temperature monitoring}

Using the diode as temperature sensor, it reports the temperature of the micro-hotplate at the MOSFETs site. This technique is based on the dependency of PIN diodes characteristics to the operating temperature biased by a stable current source (here $65 \mu \mathrm{A}$ ), the diode voltage dependency is demonstrated by Equation (1).

$V_{d}=V_{0}-d\left(T-T_{0}\right)$

with $V_{0}[\mathrm{~V}]$ the initial voltage at room temperature, $d\left[\mathrm{~V} /{ }^{\circ} \mathrm{C}\right]$ the resolution coefficient of the diode, $T\left[{ }^{\circ} \mathrm{C}\right]$ the operating temperature and $T_{0}\left[{ }^{\circ} \mathrm{C}\right]$ the room temperature. Increasing the temperature of device progressively up to $300{ }^{\circ} \mathrm{C}$ by a controlled hot chuck helped to extract the drop voltage of the diode, given by $1.2 \mathrm{mV} /{ }^{\circ} \mathrm{C}$. After this first calibration of the diode, the temperature monitoring is possible by supervising $V_{\mathrm{d}}$ variation.

\section{FAST PROTON IRRADIATION AND EFFECTS}

After applying the stabilization process, a first referential characterization is made by logging the $\mathrm{I}-\mathrm{V}$ characteristics of the MOSFETs in linear regime $\left(\mathrm{V}_{\mathrm{d}}=60 \mathrm{mV}\right)$, as well the Voltage Transfer Characteristics (VTC) of the CMOS inverter at room temperature. After that, the packaged device was removed from the test fixture box, installed in a test plate, where all the contacts are leaved in a floating condition, then fixed in front of the high flux fast protons beam facility of the Cyclotron Resource Centre (Université catholique de Louvain, Louvainla-Neuve, Belgium), pending the performing of a high proton bombardment with a fluence of $10^{14} \mathrm{p}^{+} / \mathrm{cm}^{2}$. After the irradiation process the device is reinstalled in the test fixture box for a post-irradiation characterization. The subthreshold curves of the $6 \mu \mathrm{m}$ wide MOSFET, measured in linear regime with $V_{\mathrm{ds}}=60 \mathrm{mV}$, before and after irradiation, are presented in Figure 2, where we clearly notice the wide negative shift of the post-radiation curve, in addition to the tremendous increase in the off-state leakage current. The I-V characteristics of the $24 \mu \mathrm{m}$ wide channel MOSFETs, showed a similar behavior after irradiation (see Figure 3), confirming the negative shift of the threshold voltages due to the positive trapped charges. In the other hand the VTC characteristics of the inverter showed as well a severe negative transferal, seriously changing its specific noise margin. The threshold voltage of the MOSFETs is extracted by extrapolation method in linear regime (see Table I). The irradiation dose was sufficient to change the 
parameters of MOS components, by degrading the threshold voltages of N-channel transistors with $27 \%$ and $16 \%$ for, respectively, 6 and $24 \mu \mathrm{m}$-wide channels, while the input voltage corresponding to the inversion point of the inverter was also degraded by $40 \%$ with respect to its initial value (see Figure 4).

\section{THERMAL ANNEALING}

The thermal annealing was applied after the irradiation of the devices, where the protocol consisted in increasing the temperature of the micro-hotplate by successive thermal upshots from room temperature up to $385^{\circ} \mathrm{C}$ with a total period of $16 \mathrm{~min}$. The thermal upshots were performed automatically with IC-CAP software by supplying the tungsten micro-heater with increasing voltage steps of $0.25 \mathrm{~V}$ to reach a maximum voltage of $2 \mathrm{~V}$ and requiring a maximum power consumption of $66 \mathrm{~mW}$. Each step of annealing is escorted by monitoring the corresponding temperature of the micro-hotplate.

After this annealing process, we clearly notice that the drain current versus gate voltage characteristics showed a considerable recovery, the subthreshold slope of the $6 \mu \mathrm{m}$ wide NMOSFET presented in Figure 2, is significantly decreased after the annealing and tends to establish its initial position, accordingly the off-state current is decreased, which proves the efficiency in neutralizing the trapped induced positive charges.

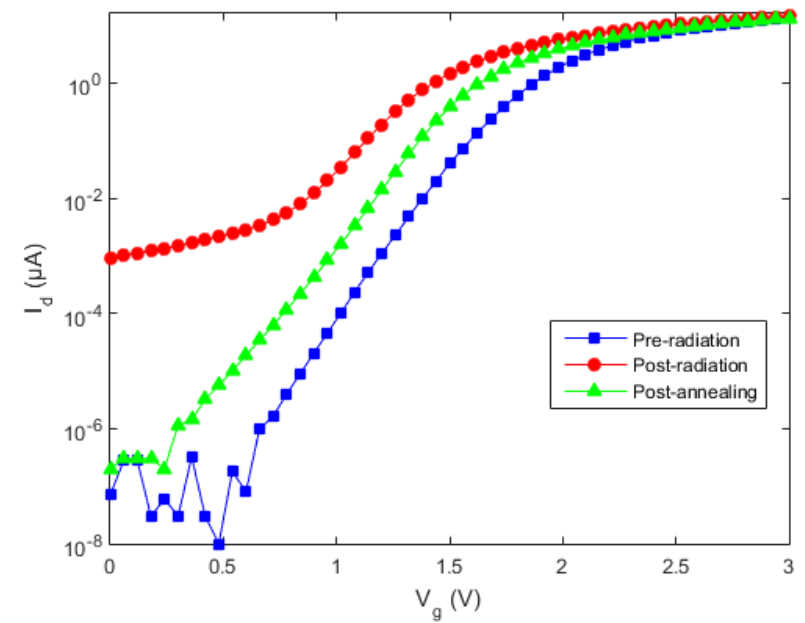

Fig. 2. Sub-threshold curves of $6 \mu \mathrm{m}$ wide channel nMOSFET in linear regime with $\mathrm{V}_{\mathrm{ds}}=60 \mathrm{mV}$, presenting its behavior before, after irradiation and after the annealing process.

The $24 \mu \mathrm{m}$ wide channel NMOSFET showed a similar behavior after the annealing process, where the I-V characteristics are significantly shifted towards the initial position (see Figure. 3). The extracted values of threshold voltage presented in Table. 1 shows the considerable recovery obtained by the annealing process and prove the reliability of this technique for the different dimensions of MOSFETs.

TABLE I

THRESHOLD VOLTAGE VARIATION

\begin{tabular}{lll}
\hline \hline & $6 \mu \mathrm{m}$ wide & $24 \mu \mathrm{m}$ wide \\
\hline$V_{\text {Th }}$ [V] Pre-radiation & 1.73 & 1.66 \\
$V_{T h}$ [V] Post-radiation & 1.25 & 1.38 \\
$V_{T h}$ [V] Post-annealing & 1.46 & 1.56 \\
\hline \hline
\end{tabular}

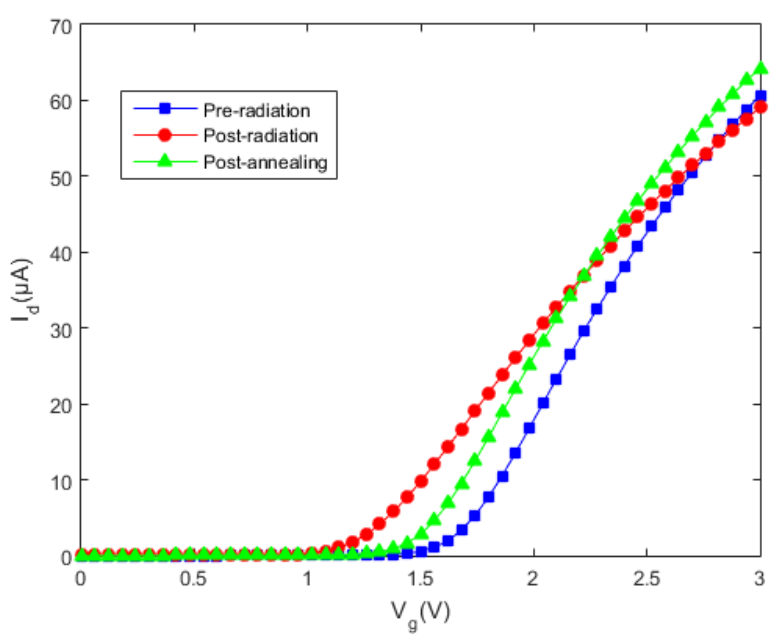

Fig. 3. Drain current versus gate voltage characteristics of $24 \mu \mathrm{m}$ wide channel $\mathrm{N}$-MOS transistor in linear regime with $\mathrm{V}_{\mathrm{ds}}=60 \mathrm{mV}$, showing the change in behavior before, after proton radiation and after thermal annealing.

After the annealing process, the percentage of the threshold voltage degradation was reduced to $15 \%$ and $6 \%$ respectively for the $6 \mu \mathrm{m}$ and $24 \mu \mathrm{m}$ wide channel transistors, while the inversion point of the single stage inverter was moved back to $24 \%$ of its initial value. Hence, the recovery of the components reached about its half.

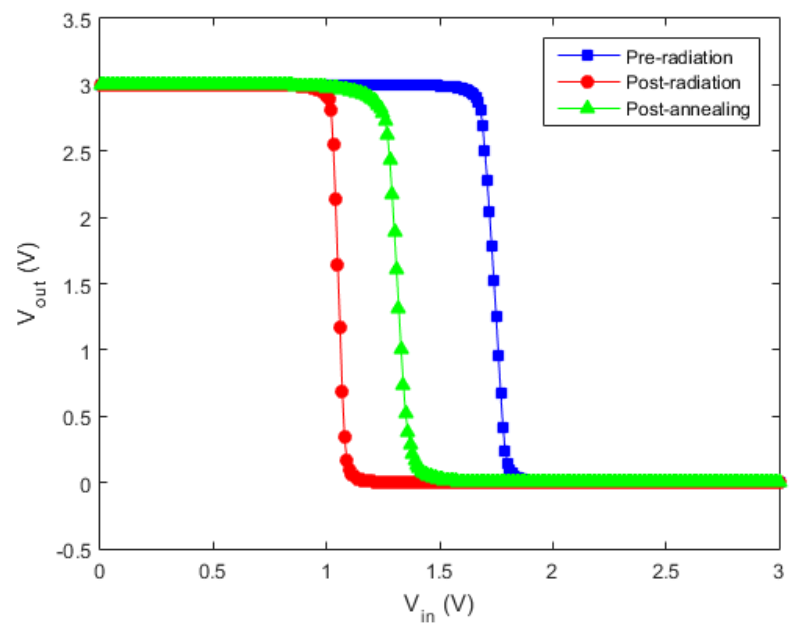

Fig. 4. Voltage transfer characteristics of the CMOS inverter presented before and after radiation and after the thermal annealing.

\section{CONCLUSION}

The $10^{14} \mathrm{p}^{+} / \mathrm{cm}^{2}$ dose of fast protons irradiation was detrimental to the electrical characteristics of the on-membrane NMOSFETs and the CMOS inverter. Thanks to the low power micro-hotplates of the special MEMS structure, a high temperature annealing up to $385^{\circ} \mathrm{C}$ was sufficient to perform a partial recovery of the threshold voltage and the off-state leakage currents of the NMOSFETs as well for the inversion point of the inverter. A total recovery of components is expected by increasing the annealing temperature. 


\section{REFERENCES}

[1] Manet P, Falmagne S, Garnier J, Berger G, Legat J-D, editors. SEE evaluation of a low-power $1 \mu \mathrm{m}$-SOI $80 \mathrm{C} 51$ for extremely harsh environments. Radiation and Its Effects on Components and Systems (RADECS), 2009 European Conference on. IEEE. 2009.

[2]Rue B, André N, Olbrechts B, Raskin J, Flandre D, editors. High Temperature SOI CMOS Low Power circuits and micro systems for MEMS cointegrated interfaces, temperature sensing and power management applications. Caneus Workshop. 2009.

[3]Rezzak N, Zhang EX, Alles ML, Schrimpf RD, Hughes H, editors. Totalionizing-dose radiation response of partially-depleted SOI devices. SOI Conference (SOI), 2010 IEEE International. IEEE. 2010.

[4] Gwyn C. Model for Radiation-Induced Charge Trapping and Annealing in the Oxide Layer of MOS Devices. Journal of Applied Physics.40(12):4886-92. 1969.

[5]Pejovic MM, Pejovic MM, Jaksic AB. Contribution of fixed oxide traps to sensitivity of pMOS dosimeters during gamma ray irradiation and annealing at room and elevated temperature. Sensors and Actuators A: Physical.174:85-90. 2012.

[6] Amor S, André N, Kilchytska V, Tounsi F, Mezghani B, Gérard P, et al. Insitu thermal annealing of on-membrane silicon-on-insulator semiconductorbased devices after high gamma dose irradiation. Nanotechnology.28(18):184001. 2017.

[7] Francis LA, André N, Gérard P, Ali SZ, Udrea F, Flandre D, editors. A lowpower and in situ annealing mitigation technique for fast neutrons irradiation of integrated temperature sensing diodes. Advancements in Nuclear Instrumentation Measurement Methods and their Applications (ANIMMA), 2015 4th International Conference on. IEEE. 2015.

[8] Amor S, André N, Gérard P, Ali S, Udrea F, Tounsi F, et al. Reliable characteristics and stabilization of on-membrane SOI MOSFET-based components heated up to $335^{\circ}$ C. Semiconductor Science and Technology.32(1):014001. 2016. 